\title{
Formación de operadores del sistema de justicia penal y cultura de la paz
}

\author{
Training of criminal justice system operators and culture of peace
}

\section{Recibido: 07-10-2021 | Aceptado: 10-12-2021}

Cristian Ortega Barrera*

* Asociación Nacional de Universidades e Instituciones de Educación Superior, ANUIES

México

\section{Resumen}

La formación de los abogados, antes de la instauración de las salidas alternas, había privilegiado la solución de los conflictos a través de un litigio, mismo que involucra la imposición de la resolución del juez sobre la voluntad de una o de ambas partes; a raíz de la Reforma Constitucional en materia de seguridad y justicia de 2008, la capacitación en el mundo jurídico ha cambiado, ahora además de solucionar el conflicto también lo entiende y ayuda a su prevención a través de mecanismos no violentos; lo que contribuye al logro de la Cultura de la Paz.

Palabras clave: Derecho penal, Educación, Cultura de Paz, Abogado, Solución de conflictos

\section{Abstract}

The training of lawyers had privileged the resolution of conflicts through litigation, which involves the imposition of the judge's resolution generating violence, because of the 2008 Constitutional Reform on Security and Justice; the training in the legal world has changed, now also solves the conflict but also understand it and prevent it through non-violent mechanisms, which contributes to the achievement of the Culture of Peace.

Key words: Criminal law, Education, Culture of Peace, Lawyer, Conflict resolutions. 


\section{INTRODUCCIÓN}

En La educación de los operadores jurídicos (jueces, defensores y fiscales y los asesores de la víctima), tradicionalmente no se ha desarrollado desde el Cultura de la Paz; sino que se les ha dotado de capacidades que son utilizadas para resolver el conflicto a través de la violencia.

Nos hemos percatado como comunidad humana que el conflicto no necesariamente es sinónimo de violencia; que se puede repensar $y$ transformar en aprendizaje que nos permita evolucionar a partir de la crisis. La promoción y educación en la Cultura de la Paz nos ayuda a alcanzar u obtener esta visión.

"La cultura de paz supone ante todo un esfuerzo generalizado para modificar mentalidades y actitudes con ánimo de promover la paz." (Torres, T. \& Corella, O., 2020, p. 5); este esfuerzo generalizado debe permear a todo tipo de actividad humana de manera que se logre no sólo la ausencia de conflictos sino también que se logre la solución de los conflictos existentes a través de soluciones no violentas.

El término Cultura de Paz, deviene de la Declaración y Programa sobre Cultural de Paz, resolución 53/243 de la Asamblea General de Naciones Unidas; al indicar que debe ser alcanzable, entre otros, a través del respeto a los derechos humanos, la solución de conflictos de forma pacífica y la igualdad; siendo que en estas labores la profesión jurídica esta presente. Este término cobra relevancia pues debemos plantearnos si en la educación jurídica se persiguen o no estos propó- sitos para poder comprender el necesario cambio en la formación de las personas estudiantes de Derecho.

En el presente escrito se explicará cómo ha sido la formación jurídica tradicional que ha privilegiado que se observe la solución de conflictos a través de la violencia y por qué la misma debiera privilegiar la formación para la Cultura de Paz, que se refleje en una mayor solución de conflictos jurídico-penales por medios pacíficos.

\section{UNA OPCIÓN AL DERECHO PENAL}

De acuerdo con la Declaración y Programa de Acción sobre una Cultura de Paz, resolución 53/243 de la Asamblea General de Naciones Unidas en su artículo 1, la Cultura de Paz es un conjunto de valores, actitudes, tradiciones, comportamientos y estilos de vida basados en ${ }^{1}$ (Naciones Unidas, 1999, Resolución 53/243, Artículo primero):

a) El respeto a la vida, el fin de la violencia y la promoción y la práctica de la no violencia por medio de la educación, el diálogo y la cooperación;

b) El respeto pleno de los principios de soberanía, integridad territorial e independencia política de los Estados y de no injerencia en los asuntos que son esencialmente jurisdicción interna de los Estados, de conformidad con la Carta de las Naciones Unidas y el derecho internacional;

c) El respeto pleno y la promoción de todos los derechos humanos y

$\overline{1}$ Negritas del Autor del presente trabajo. 
las libertades fundamentales;

d) El compromiso con el arreglo pacífico de los conflictos;

e) Los esfuerzos para satisfacer las necesidades de desarrollo y protección del medio ambiente de las generaciones presentes y futuras;

f) El respeto y la promoción del derecho al desarrollo;

g) El respeto y el fomento de la igualdad de derechos y oportunidades de mujeres y hombres;

h) El respeto y el fomento del derecho de todas las personas a la libertad de expresión, opinión e información;

i) La adhesión a los principios de libertad, justicia, democracia, tolerancia, solidaridad, cooperación, pluralismo, diversidad cultural, diálogo y entendimiento a todos los niveles de la sociedad y entre las naciones; (...)

Así entonces una Cultura de la Paz debe lograrse, promoverse y fomentarse; entre otras, a través de la práctica de la no violencia, el diálogo, el respeto a los derechos y libertades fundamentales, el arreglo pacífico de los conflictos, el respeto a la igualdad de género; y la adhesión a los principios de libertad, justicia, democracia, tolerancia, solidaridad, cooperación, pluralismo, diversidad cultural, diálogo y entendimiento a todos los niveles de la sociedad. Es por ello que la Cultura de Paz debiera permear a la sociedad en todos los niveles y desde todas las disciplinas, no obstante, y hasta hace poco tiempo, se encontraba ausente de la profesión jurídica, pero a raíz de la Reforma Constitucional en materia de seguridad y justicia de 2008, ha permeado en forma de soluciones alternas al proceso que no obstante sus bondades, en muchas ocasiones son sub utilizadas por los litigantes debido a falsas creencias derivadas de la formación del operador jurídico.

Se ha mencionado que el Derecho Penal tiene una finalidad de prevención especial y de prevención general; la prevención especial se logra cuando se ha presentado un delito y se ha castigado éste, con lo que se evita que la persona vuelva a delinquir; por otro lado, la prevención general se logra cuando entra en vigor la norma penal y con ello se evita que los ciudadanos comentan delitos; no obstante;

¿Será la norma jurídico-penal la que influye en primera instancia en el individuo para no cometer delito? Si respondiéramos afirmativamente, estaríamos sosteniendo que el ser humano pasa su vida pensando en cometer delitos, y no lo hace por temor a ser penado; ello no es así; la mayoría de los ciudadanos desarrollan su vida social observando comportamientos sustentados en reglas de conducta establecidos por la misma sociedad, los cuales tienen un trasfondo moral, ético o religioso. Estos principios son los que en primera instancia influyen en el comportamiento social. Si la mayoría de los ciudadanos no privan de la vida a otro no es por que piensen en la sanción, sino por que hay un mecanismo valorativo interior que les impide llevar a cabo esa conducta. De ahí la expresión "va contra mis principios". 
No obstante, hemos manifestado que existen ciertas conductas tan dañinas para el desarrollo de la vida social que requieren de la intervención estatal (Díaz, E. \& Roxin, C., 2017, pp. 29$30)$.

Las anteriores aseveraciones nos llevan a considerar si en la sociedad actual, ¿existen conductas que son lesivas para la vida en sociedad pero no lo suficientemente graves para ameritar la intervención estatal?, muchas de estas conductas las tenemos manifestadas en los delitos de querella pero también están manifiestas en otras formas de solución del conflicto que permiten a los involucrados una solución no impuesta por la fuerza, a diferencia de la sentencia; y que con ello se preserve la paz social por medio de la voluntad, la negociación, la comprensión mutua y la buena fé de las partes.

A raíz de la Reforma en materia de seguridad y justicia de 2008, estas nuevas formas de solución no violenta de conflictos se introducen, obligando a un cambio en la formación jurídica que transite hacia la Cultura de Paz para verla como un compromiso con la resolución pacífica de los conflictos; lo que obliga a la reflexión respecto de la "cultura de la formación" de los operadores del sistema de justicia penal para analizar, si los operadores jurídicos están preparados para esta nueva visión y nueva forma de resolución de conflictos, si el mundo de lo jurídico se ha visto enriquecido con estas formas alternas de solución y si se puede preparar a las futuras generaciones, no sólo de abogados, para facilitar el logro de la paz en pro de los derechos de las personas y de la sociedad en la búsqueda de soluciones por medios diversos a la sentencia.

\section{SÍNTESIS SOBRE LA FORMACIÓN JURÍDICA TRADICIONAL}

En el mundo de lo jurídico, la materia penal se había reservado casi de forma exclusiva para la profesión del abogado. Así entonces, cuándo los jóvenes ingresábamos a las Facultades y Escuelas de Derecho se nos instruía en el conocimiento y manejo de la ley resaltando la enseñanza respecto de las formas de solución de conflictos, pero sin indicar qué es, que integra o de dónde surge el conflicto.

Respecto de esto, se nos indicaba que la autotutela, al ser "un intento por resarcir el daño sufrido produciendo otro idéntico." (García, L., 2012, p. 17) por lo regular no estaba permitida, salvo en los casos en que el Estado no pudiera brindar protección al ciudadano ante la violación de sus derechos. Se nos enseñaba también, sobre la autocomposición como "aquella figura a través de la cual los particulares, de forma conjunta, pero sin la intervención de terceros, buscan la forma de solucionar sus diferencias a través de los acuerdos" (García, L., 2012, p. 18) al hacer referencia al desistimiento de la acción o bien a la negociación y se decía que la mejor solución para el conflicto era un buen arreglo. Pero también se hacía énfasis en la enseñanza de las reglas y procedimientos para la heterocomposición; es decir a la solución del conflicto mediante la intervención del juez que, como tercero desinteresado e imparcial, decidía quién tenía y quién no la razón, imponiendo una reso- 
lución que restituye al ganador en el uso y goce del derecho dañado; incluso mediante indemnización, ordenando también una pena para el acusado al ser declarado culpable. En pocas palabras, al tiempo que se recomendaba la negociación, no se dotaba al alumno de ninguna habilidad para desarrollarla y se enaltecía la capacidad del abogado para ganar litigios; o lo que es más, en muchas ocasiones ni siquiera se enseñaba al alumno o alumna a desarrollar un aprecio por este tipo de soluciones. El problema de la heterocomposición, como sabemos, es que en el fondo entraña una resolución que es impuesta mediante la violencia a las partes sin que se verifique si en efecto, la misma ha satisfecho los intereses de las personas involucradas o si dicha resolución las ha dejado inconformes a ambas; resolución que muchas veces no lograba resolver el conflicto en su totalidad pues no reparaba el daño producido con la conducta contraria a derecho pues si bien, se ordenaba la reparación del daño, esta no contemplaba la sanación, el perdón, la reparación del tejido social ni la reparación integral.

Esta formación, privilegiaba la violencia cultural pues se normalizaba el uso de la ley como la vía para la emisión e imposición de una resolución emitida por un órgano legitimado para ello como lo es el jurisdiccional, olvidando esta formación que la sentencia es una expresión de violencia cultural pues; "La violencia cultural hace que la violencia directa y la estructural aparezcan, e incluso se perciban, como cargadas de razón -o al menos, que se sienta que no están equivocados-" (Galtung, J., 2016, p.149)
Esta resolución impuesta por medio de la heterocomposición, en muchas ocasiones, lejos de ayudar a la resolución pacífica de los conflictos a partir de la transformación de su percepción, los perpetuaba "atacando fuego con fuego", es decir el acrecentar el conflicto en la búsqueda de una venganza institucionalizada que encontraba en la ley el instrumento para ser ejercida. Referimos esto porque se enseñaba a los futuros abogados y abogadas, a defender los derechos de las partes a toda costa, sin habernos preguntado: si ¿eso era lo que quería nuestro representado?, si con la sentencia ¿se encontró la solución de raíz al conflicto?, ¿en todos los casos era necesario elevar el conflicto a la sede judicial?, ¿las partes luego de la sentencia se sentían satisfechas? o ¿ estaban en el hartazgo del proceso que les obligó a aceptar la resolución?

Así entonces, el estudiante de Derecho y el operador del sistema de justicia penal percibían a la ley como un medio legítimo y necesario para lograr y perpetuar la paz; sin que se percibiera que el camino para llegar al arreglo del conflicto por medio de la heterocomposición, en el fondo, entraña la imposición de la decisión por medio de la violencia sin reflexionar acerca de su utilidad como mecanismo para la solución última del conflicto.

Hacía falta que la formación del abogado contemplara otras alternativas para la solución de las problemáticas al conflicto, además de que se procurara la enseñanza de la prevención pues respecto de los conflictos "No hay que esperar a que estallen, queremos trabajarlos en sus primeros estadios, o 
incluso antes de que se produzcan..." (Cascón, P., 2000, p.61); hacía falta enseñar a los profesionales del Derecho a desarrollar estrategias de gestión de las problemáticas sociales, pues si se enseñan estos mecanismos, no sólo se solucionan los conflictos existentes sino que se previenen los futuros y en cuanto surjan, se pueden implementar mecanismos de solución que eviten su escalada sin que haya necesidad del uso institucionalizado de la violencia.

Actualmente y por medio de la nueva formación que se ha dado a los operadores del sistema de justicia penal, se ha entendido que, en materia penal, no todos los conflictos ameritan la misma solución pues en la legislación actual se pueden buscar otros caminos a través del diálogo entre las partes, permitiendo el arreglo del fondo del conflicto sin generar más violencia. Esto solo acontece en los casos que se estima, no involucran la violación a un bien jurídico fundamental para la vida social como puede ser la vida, la libertad, la sexualidad, la seguridad del Estado, entre otros. Estos mecanismos se expresan en la legislación a través de los mecanismos alternativos de solución de controversias sumado al derecho de la víctima de obtener una reparación integral, que no solo comprende la reparación en términos económicos, es decir que no sólo comprende una indemnización sino también la restitución, rehabilitación, garantías de no-repetición, y las diversas medidas de satisfacción; y lo que es más, el derecho de ambas partes para el acceso a la justicia restaurativa entendida como el restablecimiento del tejido dañado con la conducta ilícita.
En esta realidad, por un lado, el mundo jurídico comenzó a percibir otras formas de solución de conflictos y abrió sus puertas a nuevos perfiles y nuevas profesiones; y por el otro comenzó a percibir que el conflicto jurídico-penal puede preverse y evitar que surja como un objetivo prioritario. En ambos casos la Cultura de la Paz se ve reflejada pues permite el diálogo que soluciona el conflicto, evita la violencia al prevenir que surja el conflicto al tiempo que vela por los Derechos Humanos de las personas involucradas. "La educación para la paz busca fomentar competencias en solucionar conflictos, en resolver guerras, en prevenir la violencia, en impedir todo aquello que es contrario a la vida y a la dignidad humana..." (Loera, E. \& Loera T., 2017, p.196)

\section{LA CULTURA DE LA PAZ Y LOS OPERADORES DEL SISTEMA DE JUSTICIA PENAL}

¿Cómo se transformó la formación de los operadores jurídicos en el sistema penal?, ¿Cómo el mundo de los jurídico se abrió a nuevos perfiles?; ¿Cómo ambas cuestiones logran la prevención de la violencia?

Hemos dicho que la percepción del operador jurídico del sistema de justicia penal ha cambiado, ahora se percibe distinto al conflicto. Ya no es sólo un choque de intereses; sino que se le ve como un proceso del que deriva un aprendizaje para las partes y para la sociedad, que puede solucionarse a través del dialogo, que puede preverse y que resulta mejor aprender a gestionarlo que a eliminarlo a través de la violencia institucionalizada en forma de sentencia. 
Para modificar la visión tradicional de la heterocomposición a la autocomposición a través de la negociación; fue necesario modificar planes y programas de estudio, no sólo a nivel universitario sino de las propias Instituciones de Justicia y comenzar la formación de nuevos perfiles en un proceso que ha tomado más de 15 años; pero que también ha exigido la transformación cultural de los formadores de dichos operadores para que a través de ellos se transmita la importancia de este tipo de soluciones alternas y de la justicia restaurativa.

Como parte de la formación de los Defensores, Ministerios Públicos, Jueces y Magistrados, y asesores jurídicos se incluyó la premisa que obliga a privilegiar, en los casos en donde sea legalmente procedente y con la voluntad de los involucrados, las salidas alternas y los mecanismos alternativos de solución de controversias; dotando a dichos operadores de las habilidades de negociación que se requieren en estos casos, pero sobre todo, de una visión que les permite tener en consideración que la pena a una conducta delictiva no necesariamente protege los intereses de las partes, que esta no siempre logra la satisfacción de los intereses de las partes pero, sobre todo, que su imposición genera violencia que impide que el tejido roto con el delito se transforme y que, además, logra en muchos casos, partes insatisfechas y una escalada de violencia. Es decir que se transformó la visión que se tenía del proceso penal para hacer de él una herramienta de prevención y de reparación efectiva e integral del daño por medio de la justicia restaurativa.
Sumado a lo anterior se les formó también en perspectiva de género y en caso de que los mecanismos alternos no prosperen o no sean procedentes, en la defensa firme del interés que representen. Sin embargo, se hace necesario ampliar el catálogo de conductas que admiten estas salidas y transformar la percepción del conflicto no sólo en el mundo jurídico sino en toda actividad permitiendo así cada vez menos procesos jurídicos penales y más salidas pacíficas al conflicto. Al hacerlo, se estará implementando un mecanismo de aprendizaje y de reconstrucción, ya que el problema con el litigio es que sólo es una forma civilizada mas no pacífica de solución del conflicto y que en el fondo no logra una prevención de mayores problemáticas. "Un estado de derecho fuerte, que protege los derechos humanos contribuye a prevenir y mitigar los delitos violentos y los conflictos, proporcionando procesos legítimos para la resolución de las reclamaciones y desincentivos para el delito y la violencia." (Torres,T. \& Corella O., 2020, p.8)

Los abogados/as también deben cambiar sus esquemas mentales no solamente para las destrezas de litigación y diseño estratégico que este sistema requiere, sino también para ayudar a sus clientes/as a lograr los objetivos que persigue el proceso penal y a cubrir, en la medida de lo posible sus necesidades (Maltos, M., 2015, p.280).

Para la formación de este capital humano en las Instituciones de Justicia se incluyeron planes de formación que mostraban a los operadores del sistema penal la importancia de dar a conocer a las personas la posibilidad de 
arreglar sus conflictos a través de los mecanismos alternativos de solución de controversias como los acuerdos reparatorios o la suspensión condicional del proceso; que hoy permiten en ciertos delitos, atacar las causas que originan el conflicto a través de una condición que es impuesta al imputado y/o a través del convenio para la reparación integral del daño, que utiliza a la mediación y la conciliación en multiplicidad de prácticas que permiten la reparación integral y la reparación del tejido social así como la importancia de voltear a ver al Derecho Penal únicamente en los casos más graves y con la conciencia de la importancia sobre el respeto a los derechos humanos de las personas involucradas en el conflicto.

En este sentido, dentro de este sistema de justicia penal reformado, la solución de conflictos no sólo se encuentra en el litigio, para esos efectos la Constitución Política de los Estados Unidos Mexicanos, en su artículo 17, indica que se preverá en la ley mecanismos alternativos de solución de controversias, así entonces para poner en marcha la operación del sistema de justicia penal acusatorio se requirió no sólo de la formación de litigantes y juzgadores, sino también de facilitadores quienes ponen en marcha estos métodos alternos $y$, quienes no necesariamente son abogados ya que por lo regular se trata de psicólogos, antropólogos, trabajadores sociales, y personas con cualquier profesión capaces de entender y dar alternativas de solución al conflicto, quienes son capaces de encontrar a través del entendimiento mutuo entre partes, soluciones consensuadas a los problemas jurídico-penales; previniendo así delitos mayores y acercando al Derecho Penal a una efectiva ultima ratio.
Estos "métodos alternos son procedimientos, a través de los cuales, de manera legal, flexible, neutral, imparcial, honesta, confidencial y equitativa, se busca una solución integral a los problemas, con ayuda de un tercero neutral." (Guerrero, S. \& Quiroz, S., 2018, s/n); ya que al lograr que las partes entiendan sus intereses, preocupaciones, puntos de vista, sentimientos y afectaciones se atiende la raíz del problema, y es desde este punto que se generan acuerdos que les permite la búsqueda de soluciones.

Así pues, por medio de este cambio en la formación, los operadores logran ver al conflicto, aún en lo penal, como una oportunidad y no como una forma de expresión de la violencia institucionalizada; ya que a través de estos mecanismos se ve un sendero diverso al proceso para llegar a la solución, solución que sólo puede conseguirse al valorar a la contraparte y su relación con ella como algo importante contribuyendo así a la restauración del tejido dañado con el delito y en el fondo al logro y mantenimiento de la paz.

Por otro lado y como otra de las aristas del mantenimiento de la paz desde la prevención estricta del delito, se capacitó al personal de algunas Instituciones encargadas de la investigación como las Fiscalías y Secretarías de Seguridad Ciudadana, entre otras; para que pudieran llevar a cabo análisis de las conductas delictivas, detectando constantes, en aras de prevenir el delito y de buscar formas más efectivas de investigación y persecución, como es el caso del Analista Criminal, quienes por lo regular son expertos en manejo de bases de datos, a través de las cuáles y por medio de análisis de in- 
teligencia, se logran detectar áreas, situaciones, horarios, etc. que están relacionados con determinadas conductas delictivas o que pueden ser causas criminógenas; así entonces se logra evitar que ocurran ilícitos por medio de acciones focalizadas en evitar y erradicar dichas causas, un ejemplo de ello serían operativos de patrullaje en ciertas zonas o en ciertos horarios, colocación de alumbrado público, creación de senderos seguros en las escuelas, etc.

Pero no sólo por medio de los mecanismos alternativos y de las acciones de prevención es que se logró el fortalecimiento de la Cultura de Paz por medio del sistema penal acusatorio; sino que también se previeron y regularon mecanismos menos agresivos de intervención para las partes. Este es el caso de la inclusión en la ley procesal penal, Código Nacional de Procedimientos Penales, de diversidad de medidas cautelares que permiten el logro del fin del proceso, al tiempo que se protege a los intervinientes y a las víctimas, sin necesidad de que el imputado vaya a prisión preventiva en todos los casos ya que, en el proceso penal anterior, el imputado por lo regular iba a prisión preventiva aún antes de saber si era o no culpable lo que incrementaba su respuesta violenta ante el sistema, con estas cautelares diversas el impacto en los derechos y en la psique de la persona procesada disminuyen, facilitando el mantenimiento de la paz.

Para el respeto de estas medidas cautelares se crearon nuevas funciones y perfiles ad hoc para la evaluación del riesgo que representa para el proceso el imputado, para la seguridad de los intervinientes o para la seguri- dad en general, así como para el seguimiento de la medida impuesta; está nueva figura, en caso de incumplimiento de la medida por el imputado, provee al Agente del Ministerio Público y al Defensor de la información necesaria para que el Juez a través de la información que le sea proporcionada por éstos; pueda decidir la medida cautelar idónea o su modificación en cualquier etapa del proceso. Estas nuevas Instituciones llamadas Unidades de Medidas Cautelares o de Servicios Previos al Juicio cuentan entre su personal con algunos abogados, trabajadores sociales, etc. quienes vigilan también las condiciones impuestas al imputado en la Suspensión Condicional del proceso; lo que contribuye también al logro y mantenimiento de la Paz en tanto duran las condiciones impuestas. Así entonces, la Cultura de Paz, ha aportado un sinfín de beneficios para el sistema penal.

\section{APORTACIONES DE LA CULTURA DE LA PAZ AL SISTEMA PENAL}

Hemos enunciado los beneficios que trajo al proceso penal y al sistema de justicia, la implementación de los mecanismos alternativos de solución de controversias en forma de nuevos procedimientos y nuevas figuras procesales; en el fondo el beneficio es el cambio cultural que permite transformar la visión del conflicto y su solución no sólo del operador jurídico del proceso, sino que de momento también han mostrado sus beneficios a las personas que han accedido a ellos; "Cuando pensamos en los conflictos, los relacionamos con la forma en que habitualmente se suelen enfrentar o 'resolver', la violencia, la anulación o destrucción de una de las partes, y no una solución justa y mu- 
tuamente satisfactoria." (Cascón, P., 2000, p.57).

La intención de la formación de estos nuevos perfiles es proporcionar al sistema las herramientas necesarias para provocar un cambio cultural. Este cambio, beneficia al sistema penal mismo y a la población en general al enterarse de estas nuevas formas de gestión del conflicto. Los nuevos perfiles formados y esta nueva forma de ver, entender, ejercer y enseñar el Derecho propiciarán una actitud distinta en el formador de manera que el proceso enseñanza-aprendizaje se vea beneficiado con nuevas formas de resolver el conflicto desde su base y con una efectiva reparación integral del daño así como de la restauración del tejido social; así entonces, la visión de venganza privada y de justicia punitiva del sistema penal se transformará en algo mucho menos violento y de mayor beneficio social que al mismo tiempo impacte en la creatividad de los profesionales al verse en la necesidad de encontrar soluciones creativas.

Ahora bien, pensar creativamente, ser creativo, actuar creativamente conlleva al mediador no sólo a pensar en nuevas formas de adaptación del medio a las necesidades de las personas para obtener una solución a una situación particular, sino también de crear un ambiente que favorezca el surgimiento de posibles soluciones de las mismas personas involucradas en la problemática. (Gorjón, F., 2020b, p. 19)

Este impacto esperado no sólo se reflejará en una baja de la violencia directa sino también de la cultural y de la estructural al permitir a las personas en conflicto un mayor acceso a la justicia y con ello, una mejor protección y un mayor respeto de sus Derechos Humanos.

Esta nueva visión del conflicto ha institucionalizado este tipo de salidas y mecanismos de prevención; se han modificado las estructuras orgánicas de los Poderes Judiciales y de las Fiscalías Generales de Justicia para poder llevar a cabo estas soluciones alternas al grado que, en el año 2018, por ejemplo, se abrieron 257 mil 154 expedientes en los Centros de Justicia Alternativa (INEGI, 2020, s/n)lo que significa el mismo número de conflictos vistos desde una perspectiva diversa al proceso penal, y que logran mayor satisfacción de las partes pues 242, 588 han sido resueltos (INEGI, 2020, s/n).

Estos mecanismos, no sólo han sido implementados en México sino también en diversas latitudes, en materia penal; tal es el caso de Bolivia, Colombia, Chile, Nicaragua, Panamá, entre otros; pero no sólo se aplican en materia penal sino también en otros campos como en México donde se desarrollan en el campo del derecho familiar o civil, por ejemplo; con el fin de construir una Cultura de la Paz.

\section{PROPUESTA PARA FACILITAR LA IMPLEMENTACIÓN DE LA CULTURA DE LA PAZ EN EL SISTEMA PENAL}

Como ha quedado señalado, la formación de los operadores del sistema de justicia penal ha permitido observar el conflicto desde otra perspectiva que los ha llevado a buscar soluciones más efectivas a éstos y 
prevenirlos. Sin embargo, queda trabajo por hacer puesto que hace falta formar para la paz y desde la paz desde las primeras trayectorias educativas de las personas; así se acostumbrará a los estudiantes, desde niños, a ver en el conflicto una oportunidad de construir y no de destruir; lo que llevará a la evolución de la sociedad. Es cada vez más frecuente que se utilicen estos mecanismos también en Instituciones Educativas, lo que sin duda contribuye a permear esta cultura, pero se requiere de mayor esfuerzo en todos los niveles educativos, no sólo a través de la resolución de conflictos y el involucramiento de la comunidad, sino también a través de la explicación e interiorización del concepto del conflicto, de las formas de solución de éste y de la importancia de la reconstrucción del tejido social; es decir, permear a todas las comunidades educativas de acciones de formación encaminadas al conocimiento de la cultura de paz y de los mecanismos alternos de solución.

Una educación que incluya la educación para la justicia, la igualdad, la solidaridad, la democracia, la libertad, el respeto a las diferencias, la diversidad cultural y, sobre todo, al desarme, a la prevención de los conflictos armados y a la transformación pacífica de los conflictos (Bahajin, S., 2018, p.94).

Para lograrlo, será necesario introducir a todos los niveles formativos y en la población en general el conocimiento y utilidad del sistema jurídico, derechos humanos, habilidades de escucha activa, para generar empatía, aspectos cívicos y éticos relacionados con la igualdad y la tolerancia, estudio so- bre el origen del conflicto, habilidades de negociación, técnicas para el manejo de la ira, de evaluación de intereses y necesidades, para ser sensibles y replantear nuestros objetivos y entender los de los demás, manejo de la violencia, perdón, etc.

Pero no podemos solamente prevenir que los conflictos futuros sean vistos y gestionados de otra forma a través de la formación de los estudiantes; si queremos observar los beneficios a corto plazo y por lo que hace a los conflictos jurídico-penales ya existentes; será necesario permitir una serie de salidas no violentas en más delitos, buscar verdaderamente al Derecho Penal como la ultima ratio; pero sobre todo, desarrollar cambio cultural para que la población y la autoridad empleen estos mecanismos de sanación en todos los procesos penales y en todos los delitos y faltas administrativas por medio de la justicia cívica; de esta forma se están previendo conflictos de mayor envergadura y se sana la relación social.

Si se quieren los anteriores objetivos, es necesario implementar campañas de difusión, que muestren el significado de la Cultura de Paz, y de las bondades de los mecanismos alternos; así la población en general acudirá a ellos con mayor confianza y con más frecuencia; aceptarán este tipo de soluciones con mayor naturalidad, dejarán de buscar al proceso penal como mecanismo de venganza para la solución a los conflictos cuando se pueden solventar por otras vías; al tiempo que se permite la valoración de la relación con el otro, la comprensión de su circunstancia y la construcción de acuerdos. 
No podemos concebir el tema de la justicia en los MASC sin considerar como elemento rector de ella a la ciudadanía, es ella el corazón de los MASC, derivado de su participación activa, como sucede en la paz positiva. Ante la importancia y trascendencia de este concepto es necesario de igual manera vincularlo al concepto de equidad ya que una de las principales características de estos métodos es que las decisiones son asumidas por las partes generando compromisos propios, auténticos y más suyos (Gorjón, F., 2015, p. 119).

Desde luego que estos cambios propuestos comprenden el mundo de la formación jurídica en donde también hace falta trabajo por hacer; se debe entender a profesión ya no sólo como la aplicación de leyes a casos concretos sino también como una fuente de comprensión del conflicto y de las formas en que el ser humano soluciona sus problemas; aumentar el entendimiento y el aprecio por otros perfiles que contribuyen en la construcción de soluciones; pero sobre todo, se debe reforzar la valoración de los mecanismos alternos de solución de conflictos a través de la modificación del perfil del abogado; donde ya no es mejor aquel que ha litigado más casos ante los Tribunales sino aquel que ha solucionado más conflictos, todo ello permitirá la aplicación de las soluciones alternas en más casos y propiciará en los representados de estos profesionales, la aceptación de estas salidas al contemplar sus bondades.

Los MASC en específico las mediaciones son consideradas herramientas para la paz, pero desde la perspectiva de su intangibilidad y su valor proactivo, es considerado un activo de la paz, desde mi perspectiva son sinónimos, porque en ambos contextos a través de su aplicación y uso provocan bienestar en las personas y en la sociedad en general, por ser un medio para el desarrollo pleno de una cultura de la no violencia, la prevención y la solución de conflictos (Gorjón, F., 2017, pp. 65-66).

Recientemente, algunas entidades como Michoacán han creado leyes para la Promoción de la Cultura de la Paz y otras las han presentado como iniciativas; incluso, se ha hablado de la presentación de una iniciativa de ley para promoverla a nivel nacional; estas adiciones legales sin duda contribuirán a la construcción de una mejor sociedad, pero dichas leyes deberán estar acompañadas de políticas públicas que las hagan realidad. Veremos en el futuro cercano qué ocurre con ellas en beneficio de la construcción de la paz que tanta falta hace al país y con ello la construcción de la felicidad como parte del ejercicio del libre albedrío en la búsqueda de soluciones.

"Nuestra capacidad de decidir es lo que nos hace felices, por lo que cuando esa capacidad se ve interrumpida o cuartada somos infelices, escoger lo que nos hace sentir bien es la clave de la felicidad" (Gorjón, F., 2020a, p.76).

\section{CONCLUSIONES}

A lo largo de la formación jurídica, se han privilegiado las formas violentas de solución del conflicto a través del proceso; estas for- 
mas imponen una solución a las partes sin verificar la efectiva satisfacción de los intereses de cada una de ellas. Estas soluciones heterocompositivas generan en el fondo violencia al ser impuestas por la autoridad y buscadas por la población como una forma de venganza.

Es necesario generar un cambio cultural que permita a la población el entendimiento y el ejercicio de la Cultura de Paz. Este cambio cultural se puede generar a través de la difusión y el empleo de mecanismos de justicia alternativa y sobre todo de justicia restaurativa que logra la solución del conflicto, pero no mediante la violencia sino mediante la voluntad, el entendimiento del otro y la verdadera satisfacción de los intereses de las partes. Estos beneficios y cambio cultural se lograrán por medio de la transformación de la profesión jurídica para ser entendida como una profesión encargada sí de la defensa de los intereses de las partes, pero además, como la encargada de generar soluciones consensuadas, apegadas al marco legal y respetuosas de los derechos humanos.

Por otro lado, la formación para la Cultura de Paz debe permear a todos los niveles educativos y entre la población en general, de forma que se acepten y apliquen cada vez más y cada vez de forma mejor, las soluciones alternas de terminación del conflicto. Sin duda los cambios en la formación jurídica y en la formación de la población en general, lograrán una mejor sociedad.

\section{TRABAJOS CITADOS}

Arango Durling, V. (2007). Paz Social y Cultura de la Paz. Panamá: Panamá Viejo.

Bahajin, S. (2018). La educación como instrumento de la Cultura de Paz. Revista Innovación educativa.

Cascón Soriano, P. (2000). Educar para el conflicto en los centros. Cuadernos de Pedagogía.

Díaz-Aranda, E., \& Roxin, C. (2017). Teoría del Delito Funcionalista. México: Flores Editor y Distribuidor.

Folberg, J., \& Taylor, A. (1996 ). Mediación. Resolución de conflictos sin litigio. México, : Limusa, Noriega Editores.

Galtung, J. (2016). La violencia: cultural, estructural y directa. Cuadernos de Estrategia. Ministerio de Defensa.

García Romero, L. (2012). Teoría General del Proceso. México, Red Tercer Milenio.

Gorjón Gómez, F. (2020b). Análisis de la Creatividad en la Mediación. Revista Ciencia Jurídica y Polífica.

Gorjón Gómez, F. (2020a). La mediación como política de bienestar. Revista Polífica, Globalidad y Ciudadanía.

Gorjón Gómez, F. J. (10, 2015). Teoría de la Impetración de la justicia. Por la necesaria ciudadanización. Comunitaria. Revista Internacional de Trabajo Social y Ciencias Sociales.

Gorjón Gómez, F. J. (2017). Mediación, su Valor Intangible y Efectos Operativos. "Una visión integradora de los métodos alternos de solución de conflictos". Ciudad de México, Tirant lo Blanch.

Guerrero Tecuanhuehue, S., \& Quiroz Villareal, S. (2018). Manual práctico de Mediación, México, Mediare A.C.

Instituto Nacional de Estadística, G. e. (2020). Censo Nacional de Procuración Justicia Estatal 2019. Resultados. México: disponible en https://www.inegi.org.mx/contenidos/programas/cnpje/2019/doc/cnpje_2019_resultados.pdf.

Loera Ochoa, E., \& Loera Ochoa, T. (2017). Un desafío para México: La cultura de la paz. Misión Jurídica.

López Garza, N. (14 de septiembre de 2020). La iniciativa de Ley General de Cultura de la Paz y Reconciliación. El Diario $M X$, págs. https://diario.mx/opinion/la-iniciati- 
va-de-ley-general-de-cultura-de-la-paz-y-reconciliacion-14-20200913-17081.

López González, M., \& Cholakian, C. (2018). Resolución Alternativa de Conflictos en América. Revista Sistemas Judiciales, disponible en htts://sistemasjudiciales.org/ wp-content/upload/2018/08/revista2.pdf.

Maltos Rodríguez, M. A. (2015). La intervención de los abogados(as) en los Mecanismos Alternativos de Solución de Controversías en Materia Penal. En F. Pérez Hurtado, S. Escamilla Cerón, G. A. Gatt Corona, O. T. Gerardo, C. de Buen Unna, E. Villalobos de González, ... et.al., Dilemas Contemporáneos sobre el Ejercicio de la Abogacía en México. México, Aba Roli, A.C.

Torres Hurtado, T. G., \& Corella Martínez, O. R. (2020). Derecho y Cultura de Paz I, Cuaderno de Trabajo. México, Telebachilleratos Comunitarios del Estado de Sonora.

UNESCO. (2019). Cultura de la Paz y No Violencia. Obtenido de https://es.unesco.org/themes/programas-construir-paz

Unidas, O. d. (1999). Resolución 53/243. Declaración del Programa de Acción Sobre una Cultura de Paz.

\section{Cristian Ortega Barrera}

Maestro y Licenciado en Derecho por la UNAM. Fue Director de Profesionalización de Projusticia de USAID/México. Litigante y docente en materia penal. Coordinador del Centro de Documentación, Investigación y Prospectiva para la Seguridad, Justicia y Paz de la ANUIES (Asociación Nacional de Universidades e Instituciones de Educación Superior). 